\title{
Design Optimisation of Multistage Depressed Collectors for High Efficiency Travelling Wave Tubes Using Genetic Algorithm
}

\author{
TK Ghosh and RG Carter \\ Lancaster University, Lancaster LA1 4YR, UK \\ Phone: +44 1524 593326, Fax: +44 1524381707 \\ e-mail: t.ghosh@lancaster.ac.uk and r.carter@lancaster.ac.uk
}

Abstract - The design of a symmetric and an asymmetric collector has been optimised using the genetic algorithm. The improvement in collector efficiency in both cases is remarkable.

\section{INTRODUCTION}

In space and air-borne applications efficiency is a prime concern for a travelling wave tube (TWT). Therefore it is always desirable to optimise the design of the multistage depressed collector (MDC) in a TWT to recover maximum power from the spent beam to achieve maximum collector and overall efficiencies. The potentials of the electrodes are first optimised to achieve maximum possible theoretical efficiency and then the geometry of the collector electrodes is optimised. A computer package has been developed using a genetic algorithm for optimisation of the design of a MDC. It is based on the 3-D simulator LKOBRA (MF) [1], the mainframe version of KOBRA3 ${ }^{1}$.

\section{OPTIMISATION OF ELECTRODE NUMBERS AND POTENTIALS}

The collector efficiency can be calculated analytically using the formula developed in [2], but this method is not suitable for optimising the electrode potentials. A computer code has been developed [3] based on the well-known hill climbing technique. In this algorithm all possible combinations of the electrode potentials are considered in such a way that the area under the spent beam curve covered by the electrodes is maximum for maximum power recovery. This makes the algorithm robust and reliable. It is also simple to implement.

Table -1 shows the improvement in collector efficiency due to the increase in the number of stages. It is observed that the improvement in collector efficiency is not significant beyond 4-stages.

\section{GENETIC ALGORITHM TO OPTIMISE THE ELECTRODE GEOMETRY}

A genetic algorithm generates a new geometry through a search procedure that uses random choice as a tool through coding of a parameter space [4]. It works from a population of strings simultaneously. Each string is a stream of binary digits with a user-specified chromosome length. The initial population of strings is created randomly. Subsequent strings are generated using three operators: (a) reproduction, (b) crossover, and (c) mutation.

In the reproduction process the strings are copied to the next generation according to the objective function or the fitness value. In our case the fitness value is the efficiency of the collector. Higher efficiency means a higher probability of contributing one or more offspring to the next generation. Once the reproduction is over the strings are entered into the mating pool.

The crossover operation is carried out into two steps. First, two parents are chosen at random. In the next step the position of crossover is chosen. If it is the $\mathrm{m}$-th position in the parent strings then all characters between $(\mathrm{m}+1)$ and 1 are swapped between the parents to create a new set of strings.

Mutation has a secondary role in the genetic algorithm and its probability is sometimes quite small. In the present case we have used the mutation operator only when the chromosomes lose vital information and all members in the population become the same.

A basic flow chart for the genetic algorithm is shown in Figure - 1. The pre-processor of this package reads the geometric data from a file and converts it into the input data file in a format suitable for KOBRA3. After each run of KOBRA3, the performance of the collector is analysed using an analysis program, which is a part of the post-processor. The collector efficiency is used as

${ }^{1}$ KOBRA 3 is distributed by INP, Junkernstrasse 99,62 Wisebaden, Germany 
the fitness parameter in the genetic algorithm that produces a new population of strings. It acts as the deciding parameter for changes in the collector geometry to be made.

This package has been used to optimise both symmetric and asymmetric collectors. The optimised collector efficiency for a 4-stage symmetric collector is about $90 \%$ and for a 2 -stage asymmetric collector it is nearly $84 \%$. The collector geometry and the trajectories for the symmetric and asymmetric collectors are plotted in Figure -2 (a) and (b) respectively. In both cases the effect of secondary electron emission has not been considered. It will be included in the future work.

Table - 1: Change in collector efficiency with the change in collector stages

\begin{tabular}{|c|c|c|c|c|c|c|}
\hline Number of Collector Stages & 1 & 2 & 3 & 4 & 5 & 6 \\
\hline Efficiency in \% & 71.5 & 81.2 & 87.2 & 89.4 & 90.3 & 91.1 \\
\hline
\end{tabular}

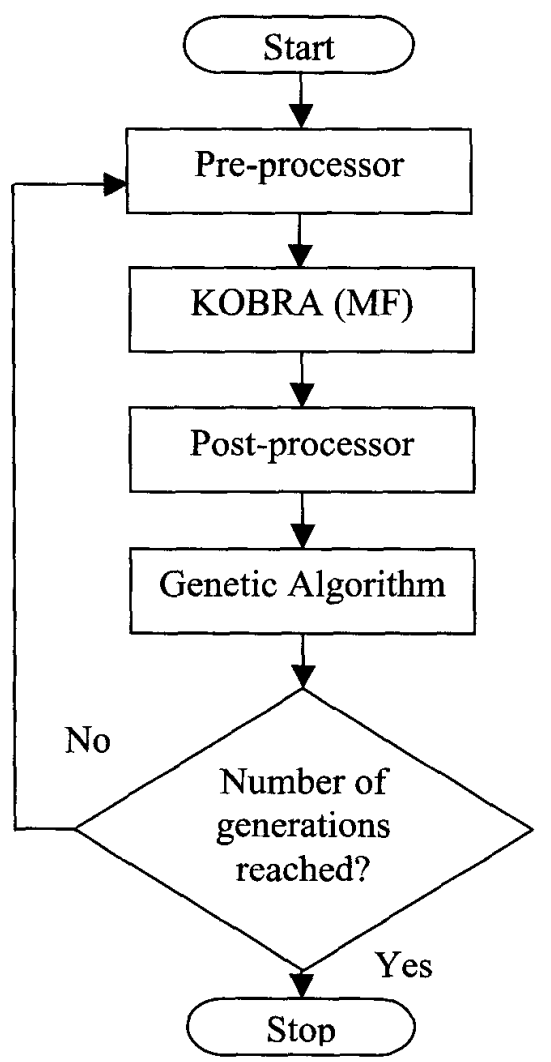

Figure - 1: Basic flow diagram of the design optimisation package

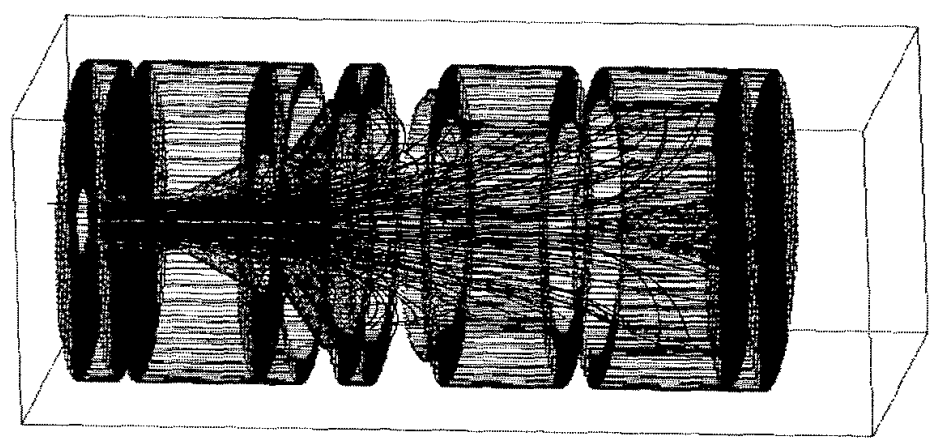

(a)

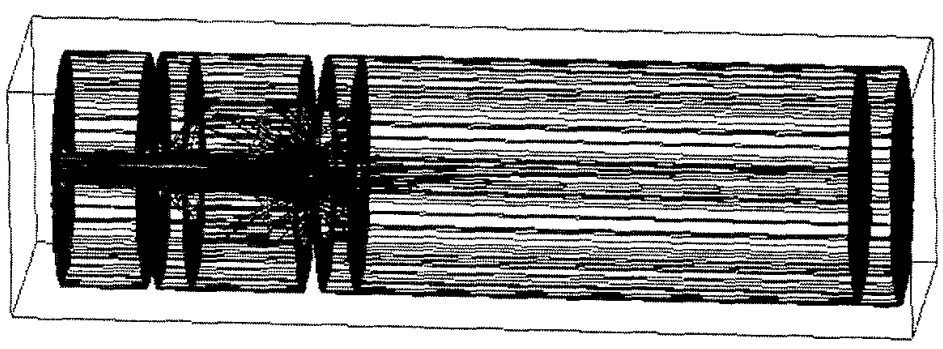

(b)

Figure - 2: Optimised geometry of (a) 4-stage symmetric collector (b) 3-stage asymmetric collector

\section{REFERENCES}

[1] TK Ghosh and RG Carter, "Improved Three Dimensional Simulation of Multistage Depressed Collectors for High Efficiency Travelling Wave Tubes," in Proc. of IVEC 2001, pp 215-220

[2] HG Kosmahl, "How to Quickly Predict the Overall TWT and the Multistage Depressed Collector Efficiency," IEEE Trans. on Electron Devices, Vol. ED-27, No. 3, pp 526-529, March 1980.

[3] TK Ghosh and RG Carter, "3-D Simulation of Multistage Depressed Collectors for High Efficiency Space TWT," Report No. MRG/2001/1, Engineering Department, Lancaster University, UK

[4] DE Goldberg, "Genetic Algorithm in Search, Optimization, and Machine Learning," AddisonWesley Publishing Company, 1989 